\title{
Modifying the Mechanics of Healing Infarcts: Is Better the Enemy of Good?
}

Samantha A. Clarke ${ }^{a}$, William J. Richardson ${ }^{a, c}$, Jeffrey W. Holmes ${ }^{a, b, c}$

\author{
Department of Biomedical Engineering ${ }^{a}$, Department of Medicine ${ }^{b}$, and \\ Robert M. Berne Cardiovascular Research Center ${ }^{c}$ \\ University of Virginia
}

\begin{abstract}
Myocardial infarction (MI) is a major source of morbidity and mortality worldwide, with over 7 million people suffering infarctions each year. Heart muscle damaged during MI is replaced by a collagenous scar over a period of several weeks, and the mechanical properties of that scar tissue are a key determinant of serious post-Ml complications such as infarct rupture, depression of heart function, and progression to heart failure. Thus, there is increasing interest in developing therapies that modify the structure and mechanics of healing infarct scar. Yet most prior attempts at therapeutic scar modification have failed, some catastrophically. This article reviews available information about the mechanics of healing infarct scar and the functional impact of scar mechanical properties, and attempts to infer principles that can better guide future attempts to modify scar. One important conclusion is that collagen structure, mechanics, and remodeling of healing infarct scar vary so widely among experimental models that any novel therapy should be tested across a range of species, infarct locations, and reperfusion protocols. Another lesson from past work is that the biology and mechanics of healing infarcts are sufficiently complex that the effects of interventions are often counterintuitive; for example, increasing infarct stiffness has little effect on heart function, and inhibition of matrix metalloproteases (MMPs) has little effect on scar collagen content. Computational models can help explain such counterintuitive results, and are becoming an increasingly important tool for integrating known information to better identify promising therapies and design experiments to test them. Moving forward, potentially exciting new opportunities for therapeutic modification of infarct mechanics include modulating anisotropy and promoting scar compaction.
\end{abstract}

\section{Keywords}

biomechanics; computational modeling; extracellular matrix; heart; myocardial infarction

\section{Address for Correspondence:}

Jeffrey Holmes, M.D., Ph.D., F.A.H.A.

Department of Biomedical Engineering, University of Virginia

Box 800759, Health System, Charlottesville, VA 22908

holmes@virginia.edu / (434) 243-6321 / (434) 982-3870 fax

http://bme.virginia.edu/holmes/index.html 


\section{Introduction}

The articles in this special issue attest to the wealth of knowledge now available regarding the biology of cardiac extracellular matrix synthesis, degradation, and remodeling, as well as the complex inflammatory and wound healing processes that occur in response to cardiac injury. Building on this knowledge, it may soon be possible to selectively manipulate the composition, structure, and mechanical properties of healing post-infarction scar tissue. Yet such therapies must be designed with particular care, because post-infarction scar plays the essential role of maintaining the mechanical integrity of the heart. Prior interventions that intentionally or accidentally modified scar structure frequently produced unexpected, even counterintuitive results, illustrating the difficulty of intervening in the complex, evolving network of biological, chemical, and mechanical interactions that govern scar structure. The goal of this review is to first outline what is known about the structure and mechanical properties of healing postinfarction scar; then to discuss how those properties affect likelihood of rupture, depression of pump function, and long-term remodeling leading to heart failure; and finally to formulate principles that could help guide the design of future interventions.

\section{Mechanical properties of healing myocardial infarcts}

Following occlusion of a coronary artery, the affected myocardium stops contracting within minutes [1] and myocytes begin to die within hours [2]. Over the next several weeks, dead myocytes are gradually removed through an inflammatory response and replaced by collagenous scar tissue generated by fibroblasts. The evolving mechanical properties of the healing infarct are an important determinant of several of the most important complications of myocardial infarction, including infarct rupture, depression of pump function of the infarcted left ventricle (LV), and LV remodeling leading to heart failure. Infarct mechanical properties appear to be determined primarily by the amount and organization of large collagen fibers; accordingly, in addition to direct mechanical testing, histologic studies of collagen structure can provide some insight into how infarct mechanical properties evolve during the natural course of healing. Available data suggest remarkable variability across animal models, complicating efforts to test interventions designed to alter infarct structure or mechanics.

\subsection{Collagen content and structure in healing infarcts}

Collagen content begins to rise 4-7 days after infarction and typically reaches a plateau by 3-6 weeks, depending on the animal model. Following coronary ligation, collagen area fraction (the fraction of a histologic section occupied by collagen) rises from 3\% to approximately $30 \%$ in the rat [3] and $60 \%$ in the pig [4] and dog [5]. Biochemical estimates of collagen content based on hydroxyproline concentration tend to be slightly lower but show a similar disparity among animal models, ranging from

$25 \%$ collagen by weight in mature rat infarcts [3,6] to $40-50 \%$ (55-70 $\mu \mathrm{g}$ hydroxyproline/mg dry weight) in dogs [7] and sheep [8]. In addition to collagen content, the degree of crosslinking of the collagen molecules can affect tissue mechanical properties. Studies of crosslinking during infarct healing suggest that crosslink density initially rises with a similar time course to collagen, but may continue to rise even after collagen content plateaus $[3,9,10]$. 
Early studies of infarct healing focused on collagen content, but the arrangement of collagen fibers within the healing infarct is also an important determinant of mechanical properties. When the heart is pressurized, the wall is under tension in the circumferential and longitudinal directions; collagen fibers in healing infarcts lie in planes parallel to the epicardium, where they resist this tension [11]. Following coronary ligation in dogs [5,12] and pigs [4], collagen fibers in the midwall are strongly aligned in the circumferential direction (Figure 1), while at the epicardium and endocardium collagen is oriented obliquely and less strongly aligned [4,5]. By contrast, Fomovsky reported that coronary ligation in rats produces structurally and mechanically isotropic infarcts in which collagen fibers at any depth are randomly oriented in the circumferentiallongitudinal plane (Figure 1) [3].

Compared to large-animal models, the standard coronary ligation model in the rat tends to create larger infarcts that involve more of the LV, including the apex. To investigate the potential influence of infarct location on scar structure, Fomovsky and colleagues used liquid-nitrogen-cooled probes to create cryoinfarcts in different locations on the rat LV [11]. They found that infarcts in different locations experienced different patterns of stretch, which correlated with the collagen fiber structure 3 weeks later. Infarcts at the apex stretched similarly in both the circumferential and longitudinal directions during healing and formed scars with randomly oriented collagen fibers; those near the equator stretched primarily in the circumferential direction and contained circumferentially aligned collagen fibers. Therefore, differences in regional mechanics during healing may explain the different collagen fiber structures reported in different animal models, although the basis for the large reported differences in collagen content remains unclear. It is also unclear whether human infarcts show similar variation in collagen fiber structure with infarct location. Further complicating the question of the relevance of various animal models to human disease, most animal work is conducted in young animals and therefore ignores potentially important effects of aging on ECM biology [13].

\subsection{Mechanical properties of healing infarcts}

There are two experimental approaches to assessing the mechanical properties of healing infarcts. One option is to excise infarct tissue and perform direct ex vivo mechanical tests. The major advantage of this approach is the ability to directly measure applied forces and resulting stretches; the disadvantage is that excision can alter mechanics due to loss of coronary perfusion pressure and/or myocyte contracture induced by cutting injury and reduced oxygenation. The alternative is to track pressures, geometry, and deformation in the intact heart, and use formulas or computational models to estimate the forces acting on the infarct. This approach maintains perfusion and viability of surviving myocardium, and allows tracking of changes over time in individual animals. However, the estimates of in situ forces depend strongly on the model employed to calculate them, and cannot be experimentally verified.

\subsubsection{In vivo deformation}

Most early studies of infarct mechanics focused on in vivo deformation. Tyberg et al. sutured strain gauges to the surface of the canine LV and plotted gauge length in the fiber direction against LV pressure [14]. Prior to occlusion, these plots traced out 
counterclockwise loops reminiscent of pressure-volume loops, with segment length increasing during diastolic filling, decreasing during ejection, and remaining nearly constant during isovolumic contraction and relaxation. Following occlusion of the coronary artery supplying the study region, the area inside the pressure-length loops rapidly decreased - reflecting the loss of active mechanical work by the muscle fibers and segments began to stretch and recoil passively along a single exponential curve. Theroux et al. extended these studies by tracking segment lengths in the infarct, border, and remote regions for 4 weeks following coronary ligation in dogs [15]. They confirmed that segmental shortening was abolished within 5 minutes of coronary occlusion and never recovered. Their data also provided some of the best early evidence of both infarct stiffening and infarct remodeling: the slope of the relationship between enddiastolic pressure (EDP) and infarct end-diastolic segment length (EDL) increased progressively over the 4 weeks of the study, while the EDL decreased by $30 \%$ beginning at 1 week.

Since these early studies, many groups have examined deformation in healing infarcts using implanted markers, sonomicrometers, ultrasound, or MRI (reviewed in [16]). Three broad themes emerge from these studies: First, following permanent coronary ligation, strains in the infarct remain close to zero throughout healing, in agreement with Theroux's original data $[3,17]$. Second, reperfusion can significantly alter regional mechanics, with circumferential and longitudinal shortening often recovering partially in reperfused infarcts [18-20]. Third, coupling to adjacent surviving myocardium can induce unexpected shears or other deformations in the infarct region that are tricky to interpret. For example, Holmes et al. measured large radial thickening strains in dense collagenous scars 3 weeks after infarction in pigs [17]. Although such strains normally indicate wall thickening due to active contraction, no surviving myocytes were apparent on histology in these scars. One additional point regarding the interpretation of in vivo strains in healing infarcts is that most studies report strains between end diastole, when muscle fibers in the heart wall are usually at their maximum length, and end systole, when they are at their minimum length. However, circumferential and longitudinal segment lengths in a passively deforming infarct typically reach their minimum length at the end of isovolumic relaxation (when LV pressure is minimum) and their maximum at the end of isovolumic contraction (when wall stress is greatest) [16]. Therefore, studies that compute strains from measurements taken only at end diastole and end systole likely neglect a substantial portion of the deformation occurring in the infarct.

\subsubsection{Ex vivo testing}

Most early ex vivo testing studies cut strips of infarct tissue and tested them uniaxially [21-23]; these early data are reviewed more extensively in [24]. However, now that it is clear that infarcts can be highly anisotropic in some cases and isotropic in others, uniaxial tests are very difficult to interpret - scar collagen fibers in any given sample could have been aligned along the test axis, aligned oblique to the test axis, or not aligned at all. Accordingly, this review focuses on the small number of tests that placed infarcts under simultaneous circumferential and longitudinal tension, better mimicking the in vivo loading state. Three studies did this by passively inflating isolated, arrested infarcted hearts while tracking deformation in the infarct. Holmes et al. found 
that 3-week-old porcine infarcts stretched as much as $40 \%$ in the longitudinal direction but less than $15 \%$ in the circumferential direction at a cavity pressure of $25 \mathrm{mmHg}$ [4]; at the same cavity pressure, peak strains in the remote myocardium were approximately $30 \%$ (longitudinal) and 35\% (circumferential). These data suggest that porcine infarcts are stiffer than undamaged passive myocardium in the circumferential but not longitudinal direction; however, it is impossible to quantify stiffness from these data without a computational model that predicts circumferential and longitudinal stress at each cavity pressure. Two passive inflation studies in rats reached different conclusions, albeit at different time points: Omens et al. measured greater infarct stretch in the circumferential direction than in the longitudinal direction in isolated arrested rat hearts 2 weeks after coronary ligation [25], while Fujimoto et al. reported equal circumferential and longitudinal infarct strains in rat hearts 8 weeks after MI [26]. Thus, consistent with the structural variability discussed in Section 2.1, the three passive inflation studies in the literature report every possible pattern of deformation in the infarct: greater longitudinal stretch, greater circumferential stretch, or equal stretch in the two directions.

Two groups have performed comprehensive biaxial mechanical testing of excised infarcts at multiple time points, and these studies also produced conflicting data. Gupta et al. studied anteroapical infarcts induced by coronary ligation in sheep and reported average circumferential and longitudinal stresses at $15 \%$ equibiaxial extension at 4 hours, 1 week, 2 weeks, and 6 weeks. They reported that longitudinal stresses were significantly greater than circumferential stresses at 1 week, while circumferential stresses were higher at 6 weeks; furthermore, stresses in both directions peaked at 1-2 weeks and then decreased, which was unexpected given that they also measured a progressive increase in collagen content with time (Figure 2) [8]. These data from Gupta are consistent with a recent report by McGarvey et al., who combined finite-element modeling with strains measured from MRI in vivo to estimate mechanical properties of healing infarcts at multiple times following infarction in pigs. McGarvey estimated increasing circumferential alignment with time following infarction, but a fiber stiffness that peaked at 1 week and declined thereafter (Figure 2) [27]. By contrast, Fomovsky et al. conducted biaxial testing of rat anteroapical infarcts induced by coronary ligation at 1 , 2,3 , and 6 weeks and found that scars were mechanically isotropic at all time points (Figure 2), and that stiffness increased monotonically with time and correlated reasonably well with collagen content on a sample-by-sample basis [3]. Yet again, these data suggest tremendous variability in infarct mechanical properties among different animal models, suggesting caution when attempting to test novel therapies in one animal model and extrapolate the results to predict outcomes in other species or in patients.

\section{Functional impact of infarct mechanical properties}

There are many different mechanisms by which a myocardial infarction can impair the pump function of the heart either directly or indirectly [24]: 1) If post-infarction necrosis weakens the infarct too much before sufficient new collagen is deposited, the heart can rupture, leading to sudden death. 2) In the first few days after infarction, the damaged region stretches passively as the rest of the heart contracts, reducing pressure generation and ejection from the LV and wasting mechanical energy. 3) Over time, as collagenous scar forms in the damaged region, systolic stretching becomes 
less problematic, but the stiff scar can limit LV filling during diastole. 4) Stretching, thinning, and outward bulging (dyskinesis) of the infarct can reduce thickening and inward motion in the borderzone due to physical coupling between the infarct and surrounding myocardium, resulting in a region of functional depression that is bigger than the actual infarct. 5) Gradual thinning of the infarct over time increases wall stress in the infarct and adjacent borderzone, which can reduce pump function and promote LV dilation. 6) In the surviving muscle, a combination of mechanical and hormonal changes due to infarction triggers hypertrophy characterized by dilation of the LV cavity with little change in wall thickness, a process that also increases wall stresses and eventually leads to dilated heart failure.

\subsection{Infarct rupture}

The first few days following infarction are characterized by myocyte necrosis and inflammation; this process involves robust secretion and activation of matrix metalloproteases (MMPs), which degrade collagen and other components of myocardial extracellular matrix (ECM) [28-30]. As a result, the infarct is mechanically weakest and most prone to rupture during this time, when degradation of pre-existing collagen and other mechanically important tissue components is underway but before significant accumulation of new collagen. Although the clinical incidence of infarct rupture is relatively low $(1-3 \%)$, it is difficult to anticipate and is associated with very high mortality rates when it occurs (60-90\% for LV freewall rupture) [31]. Accordingly, rupture accounts for $10-20 \%$ of acute deaths from myocardial infarction [31].

Most animal models of myocardial infarction display a similarly low incidence of infarct rupture; however, rupture rates following permanent left coronary artery ligation are much higher in many mouse strains. Therefore, as reviewed recently by Gao [31], current insight regarding the pathophysiology of rupture derives primarily from studies in transgenic mouse models. Broadly, these studies show that levels of inflammation and MMP expression/activation immediately following infarction are the most important contributors to infarct rupture in the mouse. For example, van den Borne reported rupture incidence ranging from $5 \%$ in BalbC mice to $62 \%$ in $129 S 6$ mice, which also contained the highest density of inflammatory cells per field 3 days post-MI of the five strains they studied [32]. Gao and colleagues showed that mouse strains that are more likely to rupture do so earlier after infarction [33] and at lower threshold infarct sizes [34]; rupture-prone strains also display higher gene expression of inflammatory mediators such as tumor necrosis factor alpha (TNF $\alpha$ ), monocyte chemoattractant protein-1 (MCP1), and interleukin-6 (IL-6) in the infarct region 3 days post-MI [34]. Confirming these correlative reports, transgenic knockout of TNF $\alpha$ [35], MMP-2 [36], and MMP-9 all reduce the risk of infarct rupture [37]. On the other hand, in some studies MMP knockout has yielded unexpected results due to the complex interactions and roles of MMPs in inflammation and healing: Ma et al. reported reduced collagen deposition and increased rupture risk following knockout of MMP-28 [38]. There is also evidence that myocardial collagen content prior to infarction can modulate rupture risk. Clinically, prior MI or LV hypertrophy appear to decrease the risk of infarct rupture [31]. Experimentally, Fang showed that insoluble collagen content (a measure of crosslinked collagen) at 4 days post-MI correlated inversely with rupture risk across multiple transgenic and nontransgenic mouse strains as well as rats; in most cases the rupture- 
resistant strains also had elevated total collagen levels even in sham-operated animals [39].

Data from transgenic mouse models can help identify factors that contribute to rupture risk, but provide little insight into the actual mechanics of the rupture process. Presumably, factors that increase rupture risk do so by weakening the tissue, making it more likely to fail at a lower stress or stretch, but the details of the relevant failure mechanism(s) remain completely unknown. In many engineering materials, a crack forms and then propagates through the material via a process that can be accurately modeled and predicted; failure stress and stiffness are independent properties of the material that arise from different aspects of its composition. By contrast, in healing infarcts the mechanism of failure is unknown, there is limited and contradictory information regarding stiffness (see Section 2.2.2) and there is even less data on failure stress. Przyklenk et al. performed uniaxial ex vivo tests of strips of canine infarcts $24 \mathrm{~h}$ after coronary ligation and found no differences in stress (tensile strength) or strain at failure compared to control tissue [23]. Connelly et al. compared uniaxial infarct tensile strength, the force required to tear out a suture threaded through the infarct, and the cavity pressure required to rupture the infarcted LV in rabbits [40]. They reported lower uniaxial tensile strength in reperfused infarcts at 1 day compared to noninfarcted myocardium, but increased tensile strength in both reperfused and non-reperfused infarcts by 5 days. Suture tearing data followed a similar trend, while whole-heart bursting tests required cavity pressures of $>400 \mathrm{mmHg}$ in all groups; the authors pointed out that based on their reported tensile strengths and burst pressures, infarct rupture should never occur in vivo. Mechanical testing of mouse infarcts is even more technically challenging. Gao et al. measured the tension required to tear short-axis rings of infarct mouse hearts and found decreased tear thresholds from 1-4 days postinfarction compared to noninfarcted controls [33], but it is difficult to compare such tear threshold values to expected in vivo wall stresses.

\subsection{Depression of left ventricular pump function}

As discussed above, within minutes after interruption of coronary flow, affected myocardium stops contracting and instead begins stretching and recoiling passively with each cardiac cycle. At the ventricular level, this results in an immediate drop in systolic function of the LV, reflected in a rightward shift in the end-systolic pressure volume relationship (ESPVR): the infarcted LV cannot eject as much blood against a given afterload, and end-systolic volume (ESV) rises [41]. Acutely, infarct size is the most important determinant of functional impairment. Sunagawa et al. found that the rightward shift of the ESPVR was linearly proportional to ischemic region size in isolated, blood-perfused canine hearts [41]. In vivo, this functional depression is more difficult to identify, because it triggers a series of compensatory mechanisms - including arterial and venous vasoconstriction, changes in heart rate and contractility in surviving myocardium, and increased diastolic pressures and sarcomere lengths - that act to restore stroke volume and mean arterial pressure. The factors that confound interpretation of common measures of LV function following MI were reviewed recently by Richardson et al. [16]. Unfortunately, ejection fraction (EF) is not only the most commonly employed metric but also the most confounded; by contrast, measuring 
cardiac output $(\mathrm{CO})$ across a range of filling pressures and comparing cardiac output curves provides the most robust assessment [16].

Although the details vary with animal model (see Section 2.2), in the weeks and months following infarction, collagen deposition and crosslinking can increase infarct stiffness dramatically. Perhaps the single most surprising finding from the past several decades of infarct mechanics studies is that even large changes in infarct stiffness have very little effect on pump function. It seems intuitively obvious that as collagen is deposited and the infarct stiffens, the infarct should stretch and bulge less, and systolic function should improve. What may be less obvious is that infarct stiffening also impairs diastolic function by reducing diastolic compliance. These effects can be difficult to measure experimentally, because infarct scar formation in vivo occurs simultaneously with LV dilation, compensatory hypertrophy, and neurohormonal changes that all modulate LV function. However, several computational models of varying complexity have all reached exactly the same conclusion: increasing infarct stiffness impairs diastolic function to a degree that almost exactly offsets systolic improvements, producing little to no shift in simulated cardiac output curves [42-44]. Studies of local surgical restraint with a variety of synthetic patches and meshes (reviewed by Clarke et al. [45] and Richardson et al. [16]) have generally reported reduced systolic and diastolic volumes without significant changes in stroke volume or cardiac output, consistent with model predictions. Importantly, these studies either assumed infarct stiffness was the same in the circumferential and longitudinal directions, or modified stiffness similarly in those two directions; the implications of infarct anisotropy on LV function are addressed in Section 4.3 below.

\subsection{Left ventricular dilation and progression to heart failure}

More than $20 \%$ of patients aged 65 or older who survive an MI will develop heart failure within 5 years [46]. The progression from myocardial infarction to heart failure is complex, but begins when the initial post-infarction drop in LV function triggers hemodynamic compensations that act to preserve arterial pressure and cardiac output [47]. Activation of the sympathetic nervous system helps preserve blood pressure through vasoconstriction and increased contractility in remote myocardium, but later triggers adverse molecular remodeling of adrenergic signaling pathways and calcium handling proteins in the heart [47]. Similarly, elevated diastolic pressures and stretch initially preserve cardiac output through the Frank-Starling mechanism, but appear to induce chamber dilation through growth and remodeling of remaining myocytes, increasing wall stresses and impairing pump function.

Infarct size is the most important determinant of acute functional depression; therefore, it is not surprising that infarct size correlates with extent of post-infarction hypertrophy, remodeling and progression to heart failure. In patients, increases in EDV and ESV are strongly correlated with infarct size, regardless of infarct location $[48,49]$. In groups of rats with different infarct sizes, LV volume and LV volume:mass ratio at matched values of LVP increased monotonically with infarct size [50], while one-year survival decreased with infarct size [51]. It is less clear whether infarct mechanical properties are an independent determinant of post-infarction LV remodeling. On the one hand, as reviewed in Section 3.2 above, modifying infarct stiffness does not improve LV function; on this basis, one might predict that modifying infarct stiffness would not alter 
the progression to heart failure. On the other hand, a few studies have reported that injecting stiff biomaterials [52] or sewing a stiff synthetic patch over the infarct region $[53,54]$ significantly reduced the extent of post-infarction LV remodeling. This finding suggests two possibilities: 1) Because infarct stiffening shifts both the end-systolic (ESPVR) and end-diastolic (EDPVR) pressure-volume relationships towards smaller volumes, it could modify mechanical stimuli that trigger myocyte hypertrophy despite the fact that it does not improve stroke volume. 2) Alternatively, expansion and thinning of the infarct region could directly account for a substantial fraction of LV dilation, in which case reducing expansion should reduce LV remodeling.

Infarct expansion was originally defined as the combination of thinning in the radial direction and dilation in the circumferential-longitudinal plane (parallel to the epicardium) [55]. The risk of infarct expansion is greatest in patients with large, transmural, anterior infarcts, and when it occurs, expansion is associated with increased risk of rupture [56], LV dilation [57,58], and heart failure [57]. Thus, therapies that prevent infarct expansion might be expected to limit adverse LV remodeling and progression to heart failure. Yet as discussed in Section 4.4 below, therapies intended to limit infarct expansion have produced mixed results in animal studies. Seeking to explain this finding, Richardson and Holmes recently reviewed quantitative studies of changes in infarct dimensions during infarct healing and found that while nearly all studies documented infarct thinning, only half found infarct expansion (an increase in circumferential or longitudinal dimensions) after the first 24 hours; many actually showed substantial circumferential and/or longitudinal compaction (Figure 3) [59]. Here again, variability among animal models and even between studies in the same animal model has potentially important consequences for the development of post-infarction therapies: the authors noted that therapies designed to prevent expansion may be effective only in the subset of cases where expansion would normally occur, potentially explaining variability in the efficacy of such therapies in animal trials.

\section{Modifying infarct mechanical properties}

For conditions discussed elsewhere in this special issue - such as heart failure with preserved ejection fraction (HFpEF) - current efforts appropriately focus on therapies to reduce fibrosis [60]. Yet the history of therapeutic interventions intended to target inflammation or fibrosis in post-infarction scar provides stark reminders that infarct scar performs an essential mechanical function, maintaining integrity of the heart wall against enormous mechanical forces. As novel, exciting ideas for modifying infarct healing continue to emerge, their development and testing should be guided by the lessons of past failures. The most important of these lessons are: 1) that novel therapies require better, broader testing for their short- and long-term chemical, mechanical, and electrical effects across a range of experimental models; and 2) that the interactions among inflammation, fibrosis, mechanical function, and electrical function are sufficiently complex that the effects of most therapies are extremely difficult to predict without appropriate computational models.

\subsection{Modifying inflammation: First, do no harm}

For investigators developing novel post-infarction therapies, early trials of postinfarction steroids provide the most sobering cautionary tale. Animal studies showing 
that post-infarction administration of steroids can reduce infarct size led to a clinical study in which steroids induced unexpected increases in infarct size and arrhythmias [61]. Follow-up studies revealed that a variety of drugs that reduce edema and inflammation, including high-dose steroids [62,63], indomethacin [64,65], and ibuprofen [66], also aggravate infarct expansion and lead to a thinner scar. Reinforcing the importance of the initial inflammatory response to post-infarction survival, BenMordechai et al. recently reported that macrophage depletion dramatically increases post-infarction mortality in mice, and that mesenchymal stem cells may influence infarct healing in part by modulating macrophage phenotype [67]. The biology of cardiac inflammation and fibrosis are discussed extensively in other articles in this special issue [68-70]. However, it remains unclear how edema and inflammation contribute to infarct mechanical properties in the first days after infarction, or exactly how their reduction leads to increased risk of rupture and arrhythmias. Given that so much remains unknown, and that basic features such as infarct rupture and arrhythmia vary so dramatically across animal models, any therapy that modulates inflammation should undergo extensive pre-clinical testing for mechanical and electrical side effects across a broad range of infarct sizes and types (reperfused vs. non-reperfused) in multiple animal models.

\subsection{Modifying composition: The important of timing}

Post-infarction collagen content is determined by a dynamic balance between production and degradation; thus, enzymes related to the synthesis and degradation of collagen are obvious potential pharmacologic targets. In particular, several groups have attempted to inhibit early collagen degradation in order to reduce infarct expansion and rupture. However, broad-spectrum MMP inhibition (MMPi) produced no significant change in collagen area fraction in mice, rats, or pigs (Figure 4) [71-73]. Similarly, transgenic knockout of MMP-3, MMP-9, MMP-12, and MT1-MMP each changed infarct collagen content $<20 \%$ compared to wild-type controls, and most of the changes were not statistically significant (Figure 4) $[37,74,75]$. Even expression of a cleavage-resistant mutant collagen did not significantly alter collagen area fraction in healing infarcts [76].

In order to better understand how so many interventions that should have altered infarct collagen content failed to do so, we constructed a simple model of collagen turnover by fitting functions describing the time course of changes in key genes/enzymes to published data [77]. We assumed that the rate of collagen production depends on fibroblast density and collagen gene expression, which peaks at 7-10 days and then declines gradually over the next several weeks [78]. We also assumed that like most enzymatic reactions, the rate of collagen degradation depends on the product of the concentration of active enzyme (MMP expression and activity rise quickly and peak just a few days after MI in rodents [79-82]) and the concentration of substrate. Clearly, this simple model omits many aspects of MMP biology that could also produce counter-intuitive effects on overall collagen content, such as changes in degradation of

other ECM components that influence collagen assembly or in the generation of peptides that influence fibrosis-related signaling [83]. Nevertheless, this simple model matched collagen area fraction data from healing rat infarcts well (Figure 4), and predicted that even $75 \%$ inhibition of MMP activity would not produce a detectable change in collagen area fraction for at least the first 3-4 weeks. Figure 4C illustrates the 
explanation suggested by the model: inhibiting MMPs early (when their levels are highest) has limited effect because there is little collagen to degrade (or spare); conversely, inhibiting MMPs later (when collagen concentration is high) has little effect because MMP concentrations have fallen to low levels. Based on this model, we hypothesize that increasing collagen production would be a more fruitful approach to enhancing infarct collagen content than inhibiting MMPs.

Interestingly, although broad-spectrum MMPi had little effect on infarct collagen content, it limited LV dilation [71,73], reduced myocyte hypertrophy in noninfarcted myocardium [72], and decreased tissue and chamber compliance [72,73], suggesting potential effects on LV remodeling independent of scar collagen turnover; studies with selective inhibitors that spare MMP-1 further confirmed this concept $[84,85]$. The fact that therapies originally intended to modify infarct collagen content proved to have greater influence on remote remodeling illustrate the enormous complexity of postinfarction healing in the heart. As an interesting parallel, a number of therapies currently employed to limit post-infarction LV remodeling appear to significantly alter infarct collagen content as an unintended side effect: angiotensin converting enzyme (ACE) inhibitors [86], angiotensin receptor blockers (ARBs) [87], and mechanical unloading [88] have all been reported to significantly decrease infarct collagen content.

\subsection{Modifying mechanical properties: Think in three dimensions}

As reviewed in Section 3.2, modeling and experimental studies have consistently shown that stiffening a healing infarct has little effect on LV pump function. Yet the models treated healing infarcts as isotropic (having the same properties in all directions), and experimental studies used interventions that probably stiffen infarcts similarly in the circumferential and longitudinal directions. When it became clear that infarcts can be highly anisotropic in some animal models, Fomovsky et al. revisited the functional impact of infarct stiffening, employing a finite-element model of an infarcted dog heart and varying not only the values but also the ratios of circumferential to longitudinal stiffness in the infarct over a wide range. They found that while stiffening the infarct equally in the circumferential and longitudinal direction had no effect on predicted stroke volume, selectively stiffening the infarct in only the longitudinal direction significantly enhanced pump function [89]. This result was unexpected, both because the stresses in the heart are greater in the circumferential direction - so that circumferential reinforcement seems intuitively more promising - and because all naturally occurring examples of anisotropic infarct scar contain circumferentially aligned collagen. Fomovsky and colleagues went on to confirm the model prediction in a large-animal experiment, demonstrating directly that reinforcing acute infarcts in only the longitudinal direction did significantly improve pump function as assessed by cardiac output curves [90]. This example suggests the power of computational models to integrate knowledge about a complex system (cardiac muscle properties, infarct mechanical properties, chamber geometry, infarct size and location, etc.) to screen potential therapies and identify the top candidates for testing.

The good news is that high-quality, well-validated models are now available for use in designing post-infarction interventions that work primarily by altering mechanics. Consistent with the work by Fomovsky discussed above, these models frequently predict outcomes that are counterintuitive, emphasizing the power of models to aid 
hypothesis generation and design of novel interventions. As examples, Wall et al. found that relatively small volumes of stiff polymers injected into the infarct border could substantially reduce wall stresses, while simulated injection of a stiff polymer into the infarct itself slightly increased wall stress [91]; Dang et al. showed that simulated surgical anterior ventricular restoration (the SAVER procedure) following infarction reduced LV size but depressed LV function, and that application of a synthetic patch exacerbated functional depression [43]; and Kerckhoffs et al. used a computational model to explore the effect of scar size and location on the ability of biventricular pacing to improve synchrony of contraction in the infarcted, dilated LV [92]. Unfortunately, comprehensive, validated models of the intracellular signaling networks and extracellular protease cascades that regulate collagen turnover in healing infarcts are not yet available to guide design of molecular interventions; however, such models are currently under development [93] and hold great promise for integrating the wealth of available molecular information reviewed elsewhere in this issue to aid in the design of post-infarction therapies.

\subsection{Modifying infarct geometry: Smaller is better}

One of the few indisputable facts about myocardial infarction is that it is better to have a small infarct than a large infarct. Thus, reducing infarct size by speeding initial revascularization has substantially reduced mortality and morbidity associated with myocardial infarction in recent years [94]. Using a similar logic, a number of studies explored the hypothesis that preventing infarct expansion would improve outcomes; yet these studies produced mixed results (see recent reviews by Gorman [95] and Clarke [45]). Richardson et al. proposed that the surprising variability in reported infarct dimension changes during healing in different animal models (Figure 3) may explain why: if only half of all infarcts normally expand, even an effective therapy would appear to work in only half of cases [59]. On the other hand, the fact that some infarcts spontaneously compact - significantly reducing their circumferential or longitudinal dimensions over time - suggests that existing mechanisms could be harnessed to reduce infarct size even after the extent of myocardial damage is determined [59]. As a promising example of this approach, Laeremanns et al. showed that a peptide blocker of the Frizzled receptors Fzd-1 and Fzd-2 increased myofibroblast density and wall thickness while decreasing in-plane infarct dimensions following infarction in mice [96]. Importantly, the peptide still exerted a significant effect when treatment was begun 2 weeks after infarction, suggesting that it promotes beneficial scar remodeling rather than simply reducing the initial extent of myocardial damage.

\section{Summary and Conclusions}

In conclusion, the data reviewed here highlight two striking facts about healing infarcts. First, collagen fiber structure (Figure 1), mechanical properties (Figure 2), and geometric remodeling (expansion/compaction and thinning, Figure 3) vary widely among different animal models, and in some cases even between different studies employing the same animal model. Unfortunately, this variability suggests that it will be extremely difficult to predict the effect of a therapy in one species based on tests in another, so that extensive testing across multiple species may be require to fully vet a new therapy. The second striking fact about the studies reviewed here is how often 
seemingly reasonable therapies - such as stiffening infarcts to improve function or inhibiting MMPs to increase scar collagen content - produced either no effect or a counterintuitive one. We propose that these frequent failures arise primarily from the complexity of the scar healing process, and the extent to which different cell types, signaling molecules, hormones, regulatory cascades, mechanical forces, and hemodynamic compensations all interact to regulate scar formation and remodeling.

In the face of this complexity, there is promising evidence that computational models can help integrate available information to predict effects of potential therapies. Models that accurately represent mechanical interactions between the healing infarct and the left ventricle have already suggested the novel concept of anisotropic infarct reinforcement (Section 4.3); similarly comprehensive, well-validated models of the molecular and signaling interactions in healing infarcts are not yet available but are under development. Finally, it is worth emphasizing that post-infarction scar plays a role essential to survival: maintaining mechanical integrity of the heart wall against the enormous forces imposed on the infarct with each beat. Thus, when developing novel therapies it is important to weigh the risks of disrupting scar structure and mechanics with the magnitude of the potential benefit the therapy can provide. On this basis, therapies such as promoting intrinsic compaction or mechanically reinforcing the infarct are not only promising in their own right, but also pose less risk of catastrophic failure than therapies that disrupt early inflammation and healing. 


\section{Acknowledgments}

The authors acknowledge funding from the National Institutes of Health (R01 HL116449 to JWH), the American Heart Association (14POST20460271 to WJR), and the National Science Foundation (NSF Graduate Research Fellowship to SAC).

\section{Disclosures}

None. 


\section{References Cited}

[1] R. Tennant, C.J. Wiggers, The effect of coronary occlusion on myocardial contraction, Am. J. Physiol. 112 (1935) 351-361.

[2] C. Connelly, W.M. Vogel, Y.M. Hernandez, C.S. Apstein, Movement of necrotic wavefront after coronary artery occlusion in rabbit., Am. J. Physiol. 243 (1982) H682-H690.

[3] G.M. Fomovsky, J.W. Holmes, Evolution of scar structure, mechanics, and ventricular function after myocardial infarction in the rat., Am. J. Physiol. Heart Circ. Physiol. 298 (2010) H221-8. doi:10.1152/ajpheart.00495.2009.

[4] J.W. Holmes, J.A. Nuñez, J.W. Covell, Functional implications of myocardial scar structure., Am. J. Physiol. 272 (1997) H2123-30.

[5] S.A. Clarke, N.C. Goodman, G. Ailawadi, J.W. Holmes, Effect of scar compaction on the therapeutic efficacy of anisotropic reinforcement following myocardial infarction in the dog., J. Cardiovasc. Transl. Res. 8 (2015) 353-61. doi:10.1007/s12265-015-9637-1.

[6] R.J. McCormick, T.I. Musch, B.C. Bergman, D.P. Thomas, Regional differences in LV collagen accumulation and mature cross-linking after myocardial infarction in rats., Am. J. Physiol. 266 (1994) H354-9.

[7] B.I. Jugdutt, R.W.M. Amy, Healing after myocardial infarction in the dog: changes in infarct hydroxyproline and topography., J. Am. Coll. Cardiol. 7 (1986) 91-102. doi:10.1016/S0735-1097(86)80265-0.

[8] K.B. Gupta, M.B. Ratcliffe, M.A. Fallert, L.H. Edmunds, D.K. Bogen, Changes in passive mechanical stiffness of myocardial tissue with aneurysm formation., Circulation. 89 (1994) 2315-26. doi:10.1161/01.CIR.89.5.2315.

[9] M.T. Vivaldi, D.R. Eyre, R.A. Kloner, F.J. Schoen, Effects of methylprednisolone on collagen biosynthesis in healing acute myocardial infarction., Am. J. Cardiol. 60 (1987) 424-425. doi:10.1016/0002-9149(87)90277-3.

[10] S.D. Zimmerman, D.P. Thomas, S.G. Velleman, X. Li, T.R. Hansen, R.J. McCormick, Time course of collagen and decorin changes in rat cardiac and skeletal muscle post-Ml., Am. J. Physiol. Heart Circ. Physiol. 281 (2001) H181622.

[11] G.M. Fomovsky, A.D. Rouillard, J.W. Holmes, Regional mechanics determine collagen fiber structure in healing myocardial infarcts, J. Mol. Cell. Cardiol. 52 (2012) 1083-1090. doi:10.1016/j.yjmcc.2012.02.012. 
[12] P. Whittaker, D.R. Boughner, R.A. Kloner, Analysis of healing after myocardial infarction using polarized light microscopy., Am. J. Pathol. 134 (1989) 879-93.

[13] M.A. Horn, A.W. Trafford, Aging and the cardiac extracellular matrix: novel mediators of fibrotic remodeling, J Mol Cell Cardiol. (2016) JMC9600.

[14] J.V. Tyberg, J.S. Forrester, H.L. Wyatt, S.J. Goldner, W.W. Parmley, H.J. Swan, An analysis of segmental ischemic dysfunction utilizing the pressure-length loop., Circulation. 49 (1974) 748-54. doi:10.1161/01.CIR.49.4.748.

[15] P. Theroux, J. Ross, D. Franklin, J.W. Covell, C.M. Bloor, S. Sasayama, Regional myocardial function and dimensions early and late after myocardial infarction in the unanesthetized dog., Circ. Res. 40 (1977) 158-65. doi:10.1161/01.RES.40.2.158.

[16] W.J. Richardson, S.A. Clarke, J.W. Holmes, Physiological implications of myocardial scar structure, Compr. Physiol. 5 (2015) 1877-1909.

[17] J.W. Holmes, H. Yamashita, L.K. Waldman, J.W. Covell, Scar remodeling and transmural deformation after infarction in the pig., Circulation. 90 (1994) 411-20.

[18] J. Bogaert, A. Maes, F. Van de Werf, H. Bosmans, M.C. Herregods, J. Nuyts, et al., Functional recovery of subepicardial myocardial tissue in transmural myocardial infarction after successful reperfusion: an important contribution to the improvement of regional and global left ventricular function., Circulation. 99 (1999) 36-43.

[19] A. Kidambi, A.N. Mather, P. Swoboda, M. Motwani, T.A. Fairbairn, J.P. Greenwood, et al., Relationship between myocardial edema and regional myocardial function after reperfused acute myocardial infarction: an MR imaging study., Radiology. 267 (2013) 701-8. doi:10.1148/radiol.12121516.

[20] C.M. Kramer, W.J. Rogers, T.M. Theobald, T.P. Power, G. Geskin, N. Reichek, Dissociation between changes in intramyocardial function and left ventricular volumes in the eight weeks after first anterior myocardial infarction., J. Am. Coll. Cardiol. 30 (1997) 1625-32.

[21] W.W. Parmley, L. Chuck, C. Kivowitz, J.M. Matloff, H.J. Swan, In vitro lengthtension relations of human ventricular aneurysms. Relation of stiffness to mechanical disadvantage., Am. J. Cardiol. 32 (1973) 889-94. doi:10.1016/S00029149(73)80153-5.

[22] C.M. Connelly, W.M. Vogel, A.W. Wiegner, E.L. Osmers, O.H. Bing, R.A. Kloner, et al., Effects of reperfusion after coronary artery occlusion on post-infarction scar tissue., Circ. Res. 57 (1985) 562-77. doi:10.1161/01.RES.57.4.562. 
[23] K. Przyklenk, C.M. Connelly, R.J. McLaughlin, R.A. Kloner, C.S. Apstein, Effect of myocyte necrosis on strength, strain, and stiffness of isolated myocardial strips., Am. Heart J. 114 (1987) 1349-1359. doi:10.1016/0002-8703(87)90536-9.

[24] J.W. Holmes, T.K. Borg, J.W. Covell, Structure and mechanics of healing myocardial infarcts., Annu. Rev. Biomed. Eng. 7 (2005) 223-53. doi:10.1146/annurev.bioeng.7.060804.100453.

[25] J.H. Omens, T.R. Miller, J.W. Covell, Relationship between passive tissue strain and collagen uncoiling during healing of infarcted myocardium., Cardiovasc. Res. 33 (1997) 351-8.

[26] K.L. Fujimoto, K. Tobita, W.D. Merryman, J. Guan, N. Momoi, D.B. Stolz, et al., An elastic, biodegradable cardiac patch induces contractile smooth muscle and improves cardiac remodeling and function in subacute myocardial infarction., J. Am. Coll. Cardiol. 49 (2007) 2292-300. doi:10.1016/j.jacc.2007.02.050.

[27] J.R. McGarvey, D. Mojsejenko, S.M. Dorsey, A. Nikou, J.A. Burdick, J.H. Gorman, et al., Temporal changes in infarct material properties: an in vivo assessment using magnetic resonance imaging and finite element simulations, Ann. Thorac. Surg. (2015). doi:10.1016/j.athoracsur.2015.03.015.

[28] M.A. Pfeffer, E. Braunwald, Ventricular remodeling after myocardial infarction. Experimental observations and clinical implications., Circulation. 81 (1990) 116172. doi:10.1161/01.CIR.81.4.1161.

[29] S. Sato, M. Ashraf, R.W. Millard, H. Fujiwara, A. Schwartz, Connective tissue changes in early ischemia of porcine myocardium: an ultrastructural study., J. Mol. Cell. Cardiol. 15 (1983) 261-75.

[30] S. Takahashi, A. Barry, S. Factor, Collagen degradation in ischaemic rat hearts., Biochem. J. 265 (1990) 233-241.

[31] X.-M. Gao, D.A. White, A.M. Dart, X.-J. Du, Post-infarct cardiac rupture: recent insights on pathogenesis and therapeutic interventions, Pharmacol. Ther. 134 (2012) 156-179. doi:10.1016/j.pharmthera.2011.12.010.

[32] S.W.M. van den Borne, V.A.M. van de Schans, A.E. Strzelecka, H.T.M. VervoortPeters, P.M. Lijnen, J.P.M. Cleutjens, et al., Mouse strain determines the outcome of wound healing after myocardial infarction., Cardiovasc. Res. 84 (2009) 273-82. doi:10.1093/cvr/cvp207.

[33] X.M. Gao, Q. Xu, H. Kiriazis, A.M. Dart, X.J. Du, Mouse model of post-infarct ventricular rupture: time course, strain- and gender-dependency, tensile strength, and histopathology, Cardiovasc. Res. 65 (2005) 469-477.

doi:10.1016/j.cardiores.2004.10.014. 
[34] X.M. Gao, Z. Ming, Y. Su, L. Fang, H. Kiriazis, Q. Xu, et al., Infarct size and postinfarct inflammation determine the risk of cardiac rupture in mice, Int. J. Cardiol. 143 (2010) 20-28. doi:10.1016/j.ijcard.2009.01.019.

[35] M. Sun, F. Dawood, W.H. Wen, M. Chen, I. Dixon, L.A. Kirshenbaum, et al., Excessive tumor necrosis factor activation after infarction contributes to susceptibility of myocardial rupture and left ventricular dysfunction, Circulation. 110 (2004) 3221-3228. doi:10.1161/01.CIR.0000147233.10318.23.

[36] S. Hayashidani, H. Tsutsui, M. Ikeuchi, T. Shiomi, H. Matsusaka, T. Kubota, et al., Targeted deletion of MMP-2 attenuates early LV rupture and late remodeling after experimental myocardial infarction., Am. J. Physiol. Heart Circ. Physiol. 285 (2003) H1229-H1235. doi:10.1152/ajpheart.00207.2003.

[37] S. Heymans, A. Luttun, D. Nuyens, G. Theilmeier, E. Creemers, L. Moons, et al., Inhibition of plasminogen activators or matrix metalloproteinases prevents cardiac rupture but impairs therapeutic angiogenesis and causes cardiac failure., Nat. Med. 5 (1999) 1135-42. doi:10.1038/13459.

[38] Y. Ma, G. V Halade, J. Zhang, T.A. Ramirez, D. Levin, A. Voorhees, et al., Matrix metalloproteinase-28 deletion exacerbates cardiac dysfunction and rupture after myocardial infarction in mice by inhibiting M2 macrophage activation., Circ. Res. 112 (2013) 675-88. doi:10.1161/CIRCRESAHA.111.300502.

[39] L. Fang, X.-M. Gao, C.S. Samuel, Y. Su, Y.L. Lim, A.M. Dart, et al., Higher levels of collagen and facilitated healing protect against ventricular rupture following myocardial infarction., Clin. Sci. (Lond). 115 (2008) 99-106. doi:10.1042/CS20070365.

[40] C.M. Connelly, S. Ngoy, F.J. Schoen, C.S. Apstein, Biomechanical properties of reperfused transmural myocardial infarcts in rabbits during the first week after infarction. Implications for left ventricular rupture., Circ. Res. 71 (1992) 401-13. doi:10.1161/01.RES.71.2.401.

[41] K. Sunagawa, W.L. Maughan, K. Sagawa, Effect of regional ischemia on the left ventricular end-systolic pressure-volume relationship of isolated canine hearts., Circ. Res. 52 (1983) 170-8. http://www.ncbi.nlm.nih.gov/pubmed/6825214.

[42] D.K. Bogen, S.A. Rabinowitz, A. Needleman, T.A. McMahon, W.H. Abelmann, An analysis of the mechanical disadvantage of myocardial infarction in the canine left ventricle., Circ. Res. 47 (1980) 728-41.

[43] A.B.C. Dang, J.M. Guccione, P. Zhang, A.W. Wallace, R.C. Gorman, J.H. Gorman, et al., Effect of ventricular size and patch stiffness in surgical anterior ventricular restoration: a finite element model study., Ann. Thorac. Surg. 79 (2005) 185-93. doi:10.1016/j.athoracsur.2004.06.007. 
[44] R.F. Janz, R.J. Waldron, Predicted effect of chronic apical aneurysms on the passive stiffness of the human left ventricle., Circ. Res. 42 (1978) 255-63. doi:10.1161/01.RES.42.2.255.

[45] S.A. Clarke, R.K. Ghanta, G. Ailawadi, J.W. Holmes, Cardiac restraint \& support following myocardial infarction, in: Cardiovascular and Cardiac Therapeutic Devices, Springer-Verlag, Berlin Heidelberg, 2014: pp. 169-206. doi:10.1007/8415_2013_163.

[46] D. Mozaffarian, E.J. Benjamin, A.S. Go, D.K. Arnett, M.J. Blaha, M. Cushman, et al., Heart disease and stroke statistics--2015 update: a report from the American Heart Association., Circulation. 131 (2015) e29-322.

doi:10.1161/CIR.0000000000000152.

[47] E. Braunwald, Heart failure, JACC Hear. Fail. 1 (2013) 1-20. doi:10.1016/j.jchf.2012.10.002.

[48] P. Chareonthaitawee, T.F. Christian, K. Hirose, R.J. Gibbons, J.A. Rumberger, Relation to initial infarct size to extent of left ventricular remodeling in the year after acute myocardial infarction, J. Am. Coll. Cardiol. 25 (1995) 567-573. doi:10.1016/0735-1097(94)00431-O.

[49] R.G. McKay, M.A. Pfeffer, R.C. Pasternak, J.E. Markis, P.C. Come, S. Nakao, et al., Left ventricular remodeling after myocardial infarction: a corollary to infarct expansion., Circulation. 74 (1986) 693-702. doi:10.1161/01.CIR.74.4.693.

[50] J.M. Pfeffer, M.A. Pfeffer, P.J. Fletcher, E. Braunwald, Progressive ventricular remodeling in rat with myocardial infarction., Am. J. Physiol. 260 (1991) H140614.

[51] M.A. Pfeffer, J.M. Pfeffer, C. Steinberg, P. Finn, Survival after an experimental myocardial infarction: beneficial effects of long-term therapy with captopril., Circulation. 72 (1985) 406-12.

[52] L.P. Ryan, K. Matsuzaki, M. Noma, B.M. Jackson, T.J. Eperjesi, T.J. Plappert, et al., Dermal filler injection: a novel approach for limiting infarct expansion., Ann. Thorac. Surg. 87 (2009) 148-55. doi:10.1016/j.athoracsur.2008.09.028.

[53] S.T. Kelley, R. Malekan, J.H. Gorman, B.M. Jackson, R.C. Gorman, Y. Suzuki, et al., Restraining infarct expansion preserves left ventricular geometry and function after acute anteroapical infarction., Circulation. 99 (1999) 135-42.

[54] S.-Y. Liao, C.-W. Siu, Y. Liu, Y. Zhang, W.-S. Chan, E.X. Wu, et al., Attenuation of left ventricular adverse remodeling with epicardial patching after myocardial infarction., J. Card. Fail. 16 (2010) 590-8. doi:10.1016/j.cardfail.2010.02.007. 
[55] G.M. Hutchins, B.H. Bulkley, Infarct expansion versus extension: two different complications of acute myocardial infarction., Am. J. Cardiol. 41 (1978) 11271132. doi:10.1016/0002-9149(78)90869-X.

[56] E.H. Schuster, B.H. Bulkley, Expansion of transmural myocardial infarction: a pathophysiologic factor in cardiac rupture., Circulation. 60 (1979) 1532-1538. doi:10.1161/01.CIR.60.7.1532.

[57] L.W. Eaton, J.L. Weiss, B.H. Bulkley, J.B. Garrison, M.L. Weisfeldt, Regional cardiac dilatation after acute myocardial infarction: recognition by two-dimensional echocardiography., N. Engl. J. Med. 300 (1979) 57-62.

doi:10.1056/NEJM197901113000202.

[58] J.A. Erlebacher, J.L. Weiss, M.L. Weisfeldt, B.H. Bulkley, Early dilation of the infarcted segment in acute transmural myocardial infarction: role of infarct expansion in acute left ventricular enlargement., J. Am. Coll. Cardiol. 4 (1984) 201-208. doi:10.1016/S0735-1097(84)80203-X.

[59] W.J. Richardson, J.W. Holmes, Why is infarct expansion such an elusive therapeutic target?, J. Cardiovasc. Transl. Res. 8 (2015) 421-430.

[60] T. McKinsey, Fibroblasts as therapeutic targets for heart failure, J. Mol. Cell. Cardiol. (2016).

[61] R. Roberts, V. DeMello, B.E. Sobel, Deleterious effects of methylprednisolone in patients with myocardial infarction., Circulation. 53 (1976) I204-6.

[62] H. Hammerman, R.A. Kloner, S. Hale, F.J. Schoen, E. Braunwald, Dosedependent effects of short-term methylprednisolone on myocardial infarct extent, scar formation, and ventricular function., Circulation. 68 (1983) 446-52. doi:10.1161/01.CIR.68.2.446.

[63] J.A. Mannisi, H.F. Weisman, D.E. Bush, P. Dudeck, B. Healy, Steroid administration after myocardial infarction promotes early infarct expansion. A study in the rat., J. Clin. Invest. 79 (1987) 1431-9. doi:10.1172/JCl112971.

[64] H. Hammerman, R.A. Kloner, F.J. Schoen, E.J. Brown, S. Hale, E. Braunwald, Indomethacin-induced scar thinning after experimental myocardial infarction., Circulation. 67 (1983) 1290-5. doi:10.1161/01.CIR.67.6.1290.

[65] H. Hammerman, F.J. Schoen, E. Braunwald, R.A. Kloner, Drug-induced expansion of infarct: morphologic and functional correlations., Circulation. 69 (1984) 611-7. doi:10.1161/01.CIR.69.3.611. 
[66] E.J. Brown, R.A. Kloner, F.J. Schoen, H. Hammerman, S. Hale, E. Braunwald, Scar thinning due to ibuprofen administration after experimental myocardial infarction., Am. J. Cardiol. 51 (1983) 877-83.

[67] T. Ben-Mordechai, R. Holbova, N. Landa-Rouben, T. Harel-Adar, M.S. Feinberg, I. Abd Elrahman, et al., Macrophage subpopulations are essential for infarct repair with and without stem cell therapy., J. Am. Coll. Cardiol. 62 (2013) 1890-901. doi:10.1016/j.jacc.2013.07.057.

[68] J. Hartupee, D.L. Mann, Role of inflammatory cells in fibroblast activation, J. Mol. Cell. Cardiol. (2016) JMC9594.

[69] N.A. Turner, Inflammatory and fibrotic responses of cardiac fibroblasts to myocardial damage associated molecular patterns (DAMPs), J. Mol. Cell. Cardiol. (2016) JMC9542.

[70] A. Papageorgiou, M. Rienks, Novel regulators of cardiac inflammation: matricellular proteins expand their repertoire, J. Mol. Cell. Cardiol. (2016) JMC9609.

[71] L.E. Rohde, A. Ducharme, L.H. Arroyo, M. Aikawa, G.H. Sukhova, A. LopezAnaya, et al., Matrix metalloproteinase inhibition attenuates early left ventricular enlargement after experimental myocardial infarction in mice., Circulation. 99 (1999) 3063-70.

[72] F.J. Villarreal, M. Griffin, J. Omens, W. Dillmann, J. Nguyen, J. Covell, Early short-term treatment with doxycycline modulates postinfarction left ventricular remodeling., Circulation. 108 (2003) 1487-92. doi:10.1161/01.CIR.0000089090.05757.34.

[73] W.M. Yarbrough, R. Mukherjee, T.A. Brinsa, K.B. Dowdy, A.A. Scott, G.P. Escobar, et al., Matrix metalloproteinase inhibition modifies left ventricular remodeling after myocardial infarction in pigs., J. Thorac. Cardiovasc. Surg. 125 (2003) 602-10. doi:10.1067/mtc.2003.197.

[74] A. Ducharme, S. Frantz, M. Aikawa, E. Rabkin, M. Lindsey, L.E. Rohde, et al., Targeted deletion of matrix metalloproteinase- 9 attenuates left ventricular enlargement and collagen accumulation after experimental myocardial infarction., J. Clin. Invest. 106 (2000) 55-62. doi:10.1172/JCl8768.

[75] G.C. Koenig, R.G. Rowe, S.M. Day, F. Sabeh, J.J. Atkinson, K.R. Cooke, et al., MT1-MMP-Dependent Remodeling of Cardiac Extracellular Matrix Structure and Function Following Myocardial Infarction, Am. J. Pathol. 180 (2012) 1863-1878. doi:10.1016/j.ajpath.2012.01.022. 
[76] M.L. Lindsey, J. Yoshioka, C. MacGillivray, S. Muangman, J. Gannon, A. Verghese, et al., Effect of a cleavage-resistant collagen mutation on left ventricular remodeling., Circ. Res. 93 (2003) 238-45. doi:10.1161/01.RES.0000085580.45279.60.

[77] W. Pilcher, W.J. Richardson, J.W. Holmes, Modeling temporal dynamics of infarct collagen turnover., Biomed. Eng. Soc. 2014 Annu. Meet., San Antonio, TX, 2014.

[78] J.P. Cleutjens, M.J. Verluyten, J.F. Smiths, M.J. Daemen, Collagen remodeling after myocardial infarction in the rat heart., Am. J. Pathol. 147 (1995) 325-38.

[79] W.C. Carlyle, A.W. Jacobson, D.L. Judd, B. Tian, C. Chu, K.M. Hauer, et al., Delayed reperfusion alters matrix metalloproteinase activity and fibronectin mRNA expression in the infarct zone of the ligated rat heart., J. Mol. Cell. Cardiol. 29 (1997) 2451-2463. doi:10.1006/jmcc.1997.0482.

[80] J.P. Cleutjens, J.C. Kandala, E. Guarda, R. V Guntaka, K.T. Weber, Regulation of collagen degradation in the rat myocardium after infarction., J. Mol. Cell. Cardiol. 27 (1995) 1281-92.

[81] J.T. Peterson, H. Li, L. Dillon, J.W. Bryant, Evolution of matrix metalloprotease and tissue inhibitor expression during heart failure progression in the infarcted rat., Cardiovasc. Res. 46 (2000) 307-15.

[82] Z.-Y. Tao, M.A. Cavasin, F. Yang, Y.-H. Liu, X.-P. Yang, Temporal changes in matrix metalloproteinase expression and inflammatory response associated with cardiac rupture after myocardial infarction in mice., Life Sci. 74 (2004) 15611572. doi:10.1016/j.lfs.2003.09.042.

[83] M.L. Lindsey, R.P. lyer, M. Jung, K. DeLeon-Pennell, Y. Ma, Matrix metalloproteinases as input and output signals for post-myocardial infarction remodeling, J. Mol. Cell. Cardiol. (2016) JMC9601.

[84] M.L. Lindsey, J. Gannon, M. Aikawa, F.J. Schoen, E. Rabkin, L. Lopresti-Morrow, et al., Selective matrix metalloproteinase inhibition reduces left ventricular remodeling but does not inhibit angiogenesis after myocardial infarction, Circulation. 105 (2002) 753-758. doi:10.1161/hc0602.103674.

[85] W.M. Yarbrough, R. Mukherjee, G.P. Escobar, J.T. Mingoia, J.A. Sample, J.W. Hendrick, et al., Selective targeting and timing of matrix metalloproteinase inhibition in post-myocardial infarction remodeling., Circulation. 108 (2003) 17539. doi:10.1161/01.CIR.0000091087.78630.79.

[86] R. Watanabe, M. Ogawa, J. Suzuki, Y. Hirata, R. Nagai, M. Isobe, A comparison between imidapril and ramipril on attenuation of ventricular remodeling after 
myocardial infarction, J. Cardiovasc. Pharmacol. 59 (2012) 323-330. doi:10.1097/FJC.0b013e3182422c1a.

[87] A. Palaniyappan, R.R.E. Uwiera, H. Idikio, B.I. Jugdutt, Comparison of vasopeptidase inhibitor omapatrilat and angiotensin receptor blocker candesartan on extracellular matrix, myeloperoxidase, cytokines, and ventricular remodeling during healing after reperfused myocardial infarction, Mol. Cell. Biochem. 321 (2009) 9-22. doi:10.1007/s11010-008-9905-3.

[88] X. Zhou, J.-L. Yun, Z.-Q. Han, F. Gao, H. Li, T.-M. Jiang, et al., Postinfarction healing dynamics in the mechanically unloaded rat left ventricle., Am. J. Physiol. Heart Circ. Physiol. 300 (2011) H1863-74. doi:10.1152/ajpheart.00830.2010.

[89] G.M. Fomovsky, J.R. MacAdangdang, G. Ailawadi, J.W. Holmes, Model-based design of mechanical therapies for myocardial infarction, J. Cardiovasc. Transl. Res. 4 (2011) 82-91. doi:10.1007/s12265-010-9241-3.

[90] G.M. Fomovsky, S.A. Clark, K.M. Parker, G. Ailawadi, J.W. Holmes, Anisotropic reinforcement of acute anteroapical infarcts improves pump function, Circ. Hear. Fail. 5 (2012) 515-522. doi:10.1161/CIRCHEARTFAILURE.111.965731.

[91] S.T. Wall, J.C. Walker, K.E. Healy, M.B. Ratcliffe, J.M. Guccione, Theoretical impact of the injection of material into the myocardium: a finite element model simulation., Circulation. 114 (2006) 2627-35. doi:10.1161/CIRCULATIONAHA.106.657270.

[92] R.C.P. Kerckhoffs, A.D. McCulloch, J.H. Omens, L.J. Mulligan, Effects of biventricular pacing and scar size in a computational model of the failing heart with left bundle branch block., Med. Image Anal. 13 (2009) 362-9. doi:10.1016/j.media.2008.06.013.

[93] A.C. Zeigler, W.J. Richardson, J.W. Holmes, J.J. Saucerman, Computational modeling of cardiac fibroblasts and fibrosis., J. Mol. Cell. Cardiol. (2016) JMC9591.

[94] H.D. White, D.P. Chew, Acute myocardial infarction., Lancet. 372 (2008) 570584. doi:10.1016/S0140-6736(08)61237-4.

[95] R.C. Gorman, B.M. Jackson, J.A. Burdick, J.H. Gorman, Infarct restraint to limit adverse ventricular remodeling., J. Cardiovasc. Transl. Res. 4 (2011) 73-81. doi:10.1007/s12265-010-9244-0.

[96] H. Laeremans, T.M. Hackeng, M.A.M.J. van Zandvoort, V.L.J.L. Thijssen, B.J.A. Janssen, H.C.J. Ottenheijm, et al., Blocking of frizzled signaling with a homologous peptide fragment of wnt3a/wnt5a reduces infarct expansion and 
prevents the development of heart failure after myocardial infarction., Circulation. 124 (2011) 1626-35. doi:10.1161/CIRCULATIONAHA.110.976969. 


\section{Figure Legends}

Figure 1. Variability in scar collagen structure following permanent coronary ligation in different animal models. A-C Histologic sections of infarct scar cut parallel to the epicardial surface at $\sim 50 \%$ transmural depth, stained with picrosirius red, and imaged under polarized light. 3-week porcine (A) and 8-week canine (B) scars contained dense, highly aligned collagen, while 3-week rat scars (C) contained randomly oriented collagen at lower density. D-F Transmural variation of mean angle (MA) and mean vector length (MVL, 0 for random alignment, 1 for perfect alignment) in the same animal models (mean $\pm S D$, replotted or unpublished data from Holmes [4], Clarke [5], and Fomovsky [3]). Porcine infarcts displayed high alignment with mean angles near circumferential across most of the wall (D). Canine infarcts had lower average alignment and more variability (larger SD) in both MA and MVL among specimens (E). Rat infarcts showed the lowest alignment $(F)$, and mean angles varied so much between specimens that the fiber distributions were statistically isotropic (no preferred direction).

Figure 2. Variability in mechanical behavior of myocardial infarct scar following permanent coronary ligation in different animal models. A Gupta et al. performed ex vivo testing of healing sheep infarcts and found that stresses at $15 \%$ equibiaxial stretch peaked at 2 weeks, with significant anisotropy present at some time points but not others [8]. B McGarvey et al. estimated scar mechanical properties following coronary ligation in pigs by fitting a finite-element model to in vivo data; simulated $5 \%$ equibiaxial stretch using their fitted parameters predicted peak stresses and anisotropy at 1 week [27]. C Fomovsky et al. found that healing rat scars were isotropic at all time points; simulated $5 \%$ equibiaxial stretch using their fitted parameters produced increasing stresses as healing progressed [3].

Figure 3. Geometric remodeling of post-infarction scars across a range of species (data replotted from Richardson et al. [59]). A Quantitative studies of chronic changes in infarct thickness almost always find significant thinning, regardless of animal model or time point (box plots show range, $25 \%$ ile, mean, and $75 \%$ ile of values reported at least 1 week after $\mathrm{MI}$, normalized to dimensions $3 \pm 2$ days post-MI). B By contrast, quantitative measurements of in-plane (circumferential or longitudinal) dimensions show a mix of expansion, spontaneous compaction, or no significant change.

Figure 4. Effect of MMP inhibition and knockout on scar collagen content. A Reported collagen area fractions (AF) for several studies of MMP inihibition (circles) or knockout (squares), plotted with control (open symbol) and matching intervention (filled symbol) connected by a solid line; see text for details. B An ordinary differential equation model (black solid line, see text) matches AF from healing rat infarcts (circles, mean $\pm S D$ ), and predicts that $75 \%$ inhibition of MMPs would increase collagen content by less than 1 SD at 3 weeks. C The key feature of the model is that the rate of collagen degradation (dotted line) is proportional to the product of enzyme (MMP) and substrate (collagen) concentrations, which peak at different times, keeping net degradation rate relatively low. 

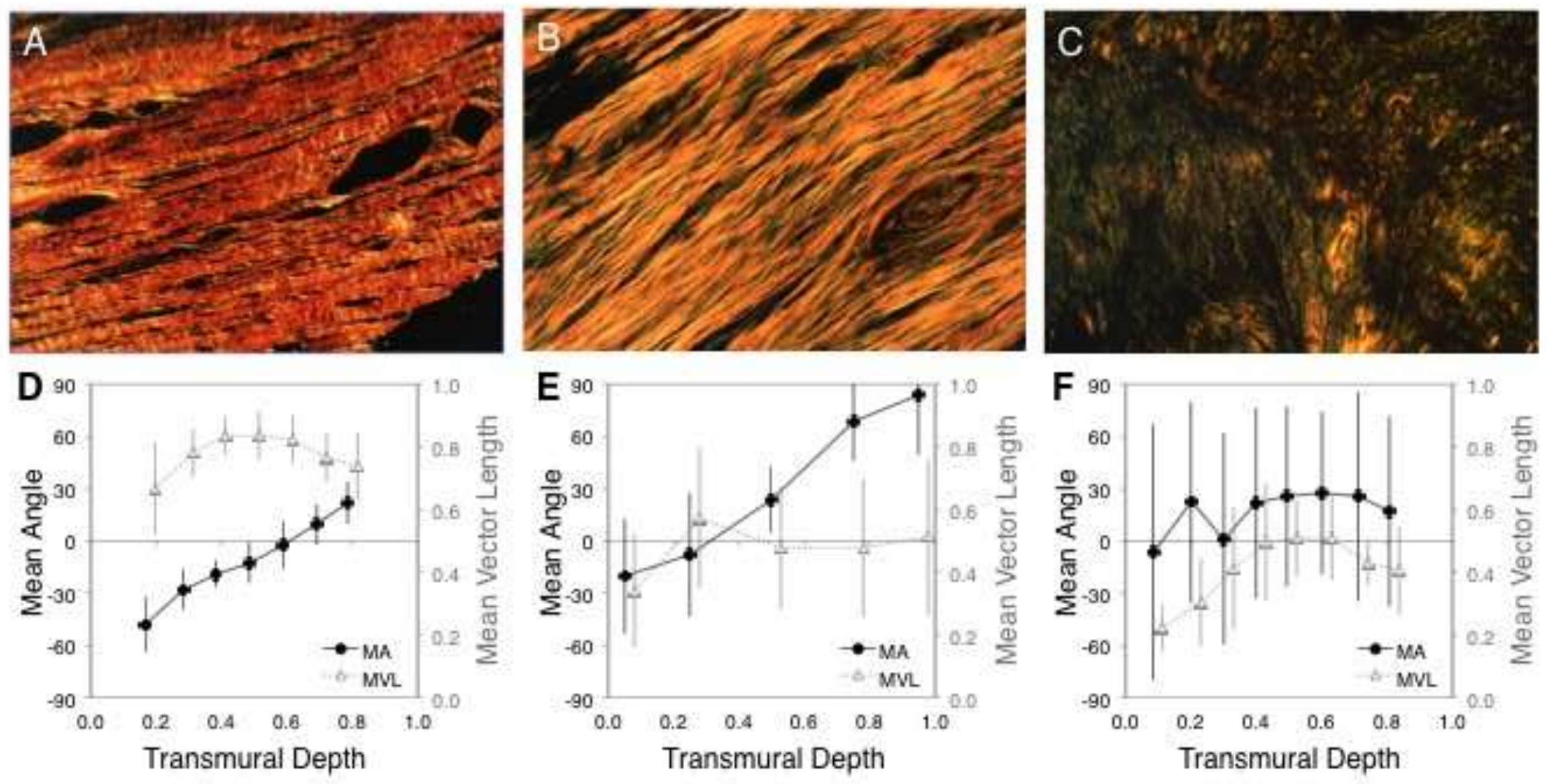

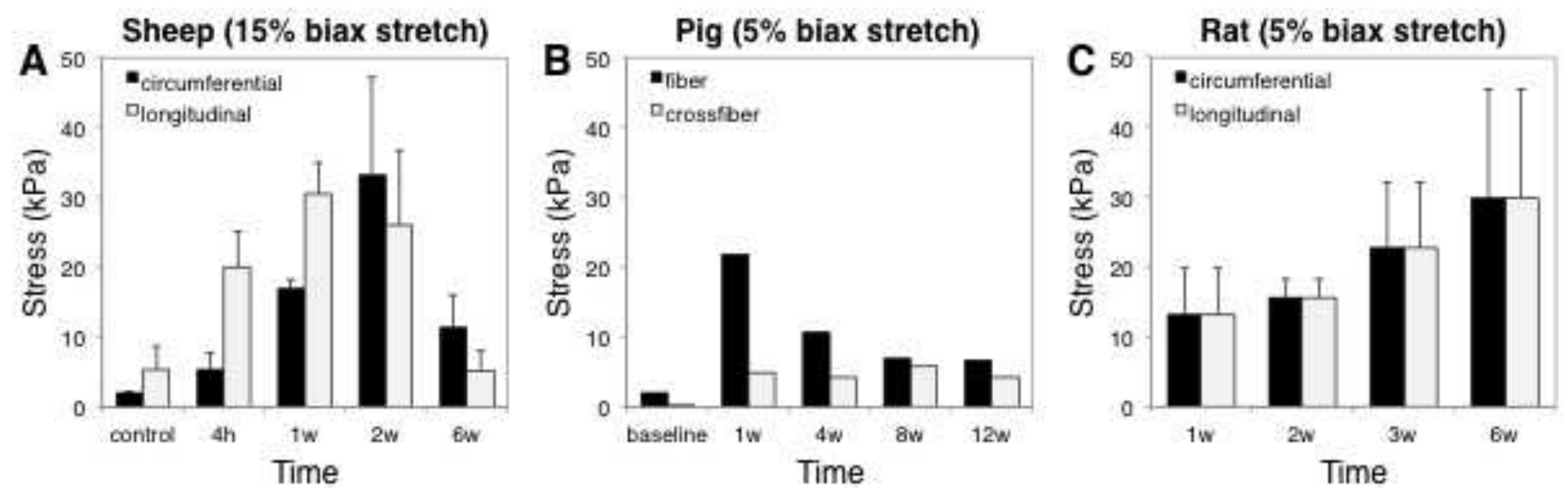

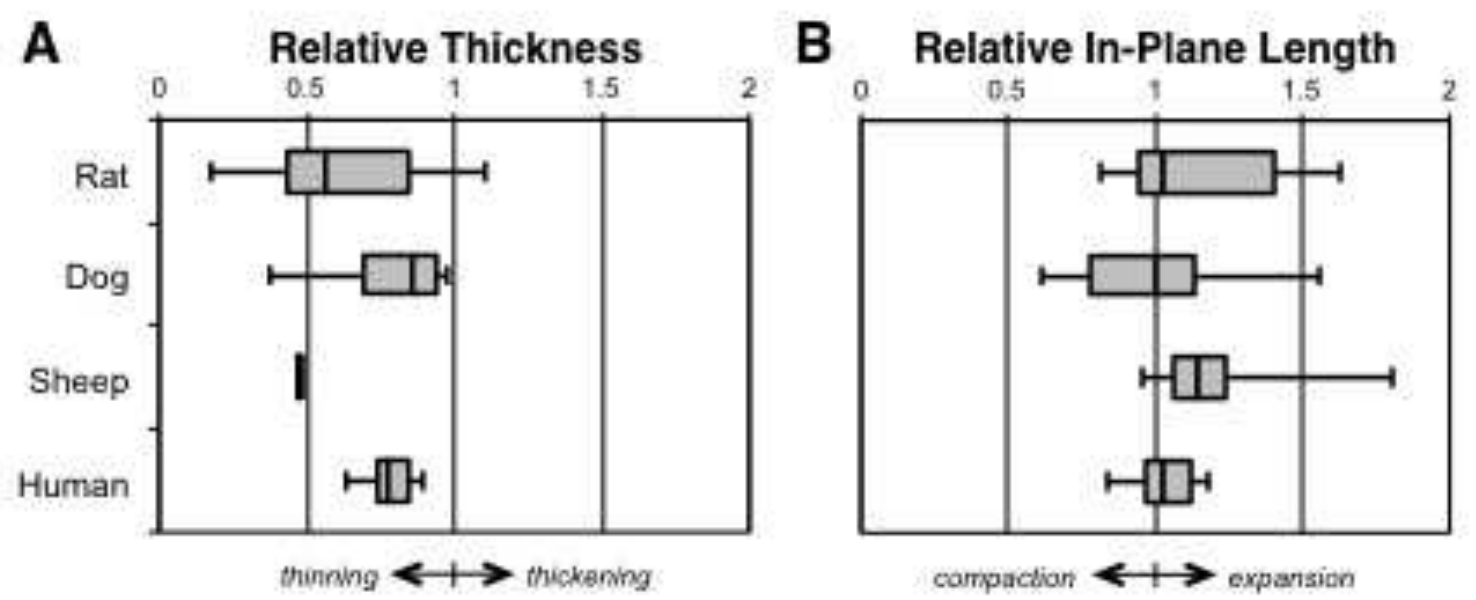

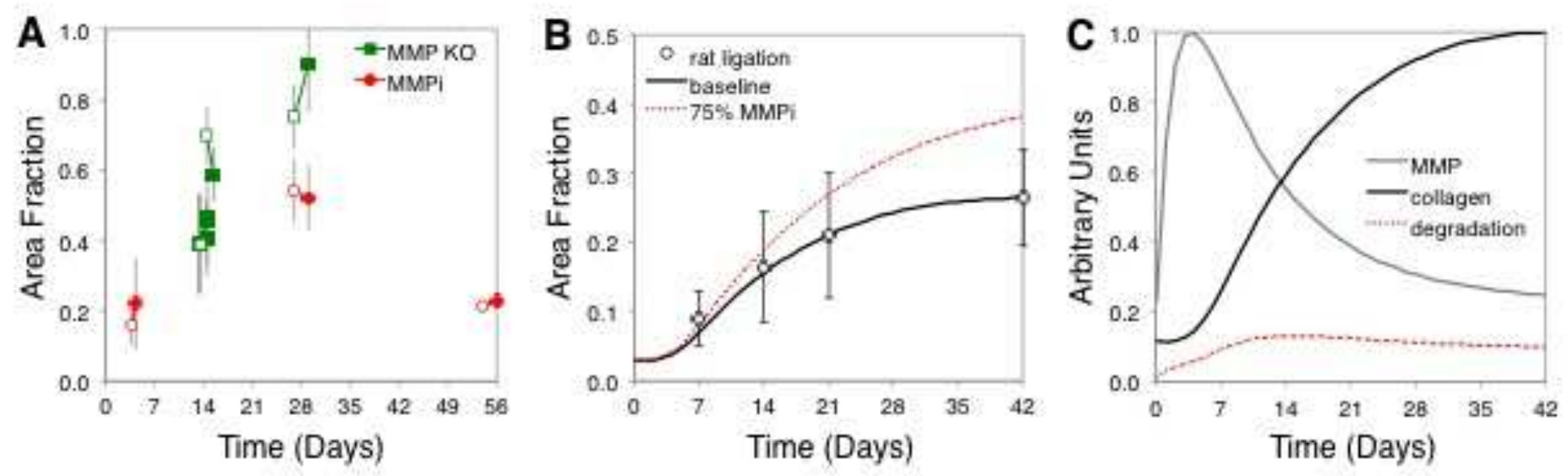